\title{
Depression symptoms reduce physical activity in COPD patients: a prospective multicenter study
}

\author{
This article was published in the following Dove Press journal: \\ International Journal of COPD \\ 10 June 2016 \\ Number of times this article has been viewed
}

\section{Iván Dueñas-Espín ${ }^{1-5}$ \\ Heleen Demeyer 6 \\ Elena Gimeno-Santos ${ }^{1-3}$ \\ Michael I Polkey ${ }^{7}$ \\ Nicholas S Hopkinson ${ }^{7}$ \\ Roberto A Rabinovich ${ }^{8}$ \\ Fabienne Dobbels 9 \\ Niklas Karlsson ${ }^{10}$ \\ Thierry Troosters ${ }^{6,11}$ \\ Judith Garcia-Aymerich ${ }^{1-3}$ \\ On behalf of the PROactive Consortium}

'ISGlobal, Centre for Research in Environmental Epidemiology (CREAL), ${ }^{2}$ Universitat Pompeu Fabra (UPF),

${ }^{3}$ CIBEREpidemiología y Salud Pública (CIBERESP), Barcelona, Spain; ${ }^{4}$ Secretaría Nacional de Educación Superior, Ciencia, Tecnología e Innovación del Ecuador (SENESCYT), Quito, Ecuador; ${ }^{5}$ Institut d'investigacions Biomèdiques August Pi i Sunyer (IDIBAPS), Barcelona, Spain; ${ }^{6}$ Department of Rehabilitation Sciences, KU Leuven, Leuven, Belgium; ${ }^{7} \mathrm{NIHR}$ Respiratory Biomedical Research Unit at the Royal Brompton and Harefield NHS Foundation Trust and Imperial College, London, UK; ${ }^{8}$ ELEGI Colt Laboratory, Centre for Inflammation Research, The Queen's Medica Research Institute, University of Edinburgh, Edinburgh, UK; ' Department of Public Health and Primary Care, Academic Centre for Nursing and Midwifery, KU Leuven, Leuven, Belgium; ${ }^{10} \mathrm{Health}$ Economics and Outcomes Research, AstraZeneca R\&D, Mölndal, Sweden; "Department of Respiratory Diseases, University Hospitals Leuven, Leuven, Belgium

Correspondence: Judith Garcia-Aymerich Centre for Research in Environmental Epidemiology, Doctor Aiguader 88, 08003 Barcelona, Spain

Tel +34 932147350

Fax +34 932147302

Email jgarcia@creal.cat
Background: The role of anxiety and depression in the physical activity (PA) of patients with COPD is controversial. We prospectively assessed the effect of symptoms of anxiety and depression on PA in COPD patients.

Methods: We evaluated anxiety and depression (Hospital Anxiety and Depression Scale [HADS]), PA (Dynaport ${ }^{\circledR}$ accelerometer), and other relevant characteristics in 220 COPD patients from five European countries at baseline and at 6 and 12 months of follow-up. HADS score was categorized as: no symptoms (score $0-7)$, suggested $(8-10)$, and probable $(>11)$ anxiety or depression. We estimated the association between anxiety and depression at $t$ (baseline and 6 months) and PA at $t+1$ (6 and 12 months) using regression models with a repeated measures approach.

Results: Patients had a mean (standard deviation) age of 67 (8) years, forced expiratory volume in 1 second $57(20) \%$ predicted. At baseline, the prevalence of probable anxiety and depression was $10 \%$ and $5 \%$, respectively. In multivariable models adjusted by confounders and previous PA, patients performed 81 fewer steps/day ( $95 \%$ confidence interval, -149 to $-12, P=0.02$ ) per extra point in HADS-depression score. HADS-anxiety symptoms were not associated with PA.

Conclusion: In COPD patients, symptoms of depression are prospectively associated with a measurable reduction in PA 6 months later.

Keywords: COPD, anxiety, depression, HADS, physical activity, prospective study

\section{Introduction}

The determinants of physical activity (PA) in patients with COPD are poorly understood. A recent systematic review identified several clinical, functional, sociodemographic, and lifestyle factors associated with PA in these patients, but the quality of evidence was low to very low, mainly due to the use of a cross-sectional design and lack of control for confounders. This lack of information is in contrast with the importance of PA in COPD, as regular PA has been consistently related to a reduced risk of hospitalizations and death. ${ }^{1}$

Among the potential determinants of PA that can be modified at the clinical setting, anxiety and depression are relevant because they are highly prevalent in COPD patients and affect COPD prognosis. ${ }^{2-4}$ Anxiety has been reported in $\sim 40 \%$ of COPD patients. ${ }^{5}$ It can lead to tachypnea, which may worsen lung hyperinflation, leading to increased exertional dyspnea, reduced exercise capacity, and poor quality of life. ${ }^{6}$ Depression, in turn, has been identified in $\sim 25 \%$ of COPD patients ${ }^{5}$ and is also associated with poorer exercise capacity and worse health status. ${ }^{2}$

Previous studies have assessed the association between anxiety and depression symptoms with PA. ${ }^{7-15}$ However, they have a cross-sectional design so the directionality of the association (eg, whether anxiety/depression reduces PA or 
PA reduces anxious/depressive symptoms) could not be established. Moreover, prior analyses have not considered the role of potentially relevant confounders, like exercise capacity $^{9,11,12,15,16}$ or smoking status. ${ }^{7-11,13}$ Hence, it remains unclear if anxiety or depression exerts an independent effect on PA levels alongside other factors commonly influencing these levels. Finally, most studies mentioned ${ }^{7-9,12,14,15}$ used indirect measures of PA such as questionnaires, so there is potential for misclassification of the primary outcome of interest (ie, PA). ${ }^{17}$

We therefore aimed to assess the effect of anxiety and depression symptoms on PA in patients with COPD, overcoming the limitations of previous studies by using a prospective design, measuring PA with an accelerometer and controlling for a wide range of potential confounders. We hypothesized that symptoms of anxiety and depression are inversely related with future PA in COPD patients. This study is part of the "Physical Activity as a Crucial Patient Reported Outcome in COPD (PROactive)" project.

\section{Methods}

See complete version in the Supplementary material.

\section{Design}

Prospective study, with repeated measures at baseline, 6 months, and 12 months. Clinical trial registration: www. clinicaltrials.gov; number: NCT01388218.

\section{Study population}

Between July 12th and November 18th, 2011, we recruited a consecutive sample of 236 patients with COPD defined by spirometry (post-bronchodilator forced expiratory volume in 1 second to forced vital capacity ratio $\left[\mathrm{FEV}_{1} / \mathrm{FVC}\right]<0.70$ ), and followed them for 1 year with visits at baseline and at 6 and 12 months. Patients were recruited from tertiary hospitals, rehabilitation centers, and primary care settings from Athens (Greece), Edinburgh and London (UK), Leuven (Belgium), and Groningen (the Netherlands). Inclusion criteria were: i) men or women at least 40 years of age, ii) with medical diagnosis of COPD $\left(\mathrm{FEV}_{1} / \mathrm{FVC}\right.$ less than $\left.70 \%\right)$ confirmed by spirometry, iii) smoking history equivalent to at least 10 pack years, and iv) able to read and write and to use electronic devices and PA monitors. Exclusion criteria were: i) orthopedic, neurological or other complaints that significantly impair normal biomechanical movement patterns, ii) respiratory diseases other than COPD (eg, asthma), and iii) cognitive impairment; further details on inclusion and exclusion criteria have been reported elsewhere. ${ }^{18}$ For the current study we additionally excluded patients who at baseline had a clinical diagnosis and/or active treatment for anxiety or depression $(n=16)$. Thirty-seven patients were lost to follow-up; there were no baseline differences between patients followed and lost to follow-up except for a lower frequency of cardiovascular diseases in those lost to follow-up $(32.4 \%$ vs $57.9 \%, P$-value $=0.05)$ (Table S1). No patients were diagnosed with anxiety or depression during follow-up.

This study was conducted in accordance with the amended Declaration of Helsinki, advised and approved by the PROactive ethics and patient advisory boards, approved by local institutional review boards (Commissie Medische Ethiek van de Universitaire Ziekenhuizen KU Leuven; Medische Ethische Toetsingscommissie Universitair Medisch Centrum Groningen; Lothian Ethics Committee; South East Scotland Research Ethics Committee; Scientific Council of the General Hospital for Chest Diseases, Sotiria), and written informed consent was obtained from all patients.

\section{Measurements}

At baseline, we collected age, sex, ethnicity, marital status, living status, socio-economic status, and working status. Anxiety and depression symptoms were evaluated at baseline and at 6 months, using the linguistically and culturally validated versions of the Hospital Anxiety and Depression Scale $(\mathrm{HADS})^{19}$ that provides a HADS-depression (HADS-D) and a HADS-anxiety (HADS-A) score. Both total scores range from 0 to 21. According to literature in the field values from 0 to 7 are considered normal, a score of 8 to 10 is suggestive of the presence of the corresponding mood disorder, and a score of 11 or higher indicates probable presence of the corresponding mood disorder. ${ }^{20}$

PA was measured at baseline, and at 6 and 12 months (mean [standard deviation, SD]) lapse 177 (12) days between baseline and 6 months visits, and 179 (14) days between 6 and 12 months visit, using a validated accelerometer, ${ }^{21,22}$ the Dynaport MoveMonitor (McRoberts BV, The Hague, the Netherlands). Patients were instructed to wear it at least 10 hours per day during 7 days. We defined an a priori valid activity monitor measurement if patients recorded at least 3 days per week (not necessarily consecutive) with at least 10 hours per day, without distinction between weekdays and weekends, according to previous literature. ${ }^{23-25} \mathrm{We}$ obtained the following variables: wearing time, steps per day, and time in locomotion.

We collected additional information at baseline and at 6 months as detailed in the Supplementary material, briefly, smoking history, COPD exacerbations history, dyspnea, quality of life (COPD assessment test [CAT]), doctor's diagnosis of comorbidities, treatments, body mass index (BMI), fat free 
mass index, lung function $\left(\mathrm{FEV}_{1}, \mathrm{FVC}\right.$, total lung capacity [TLC], residual volume [RV], RV/TLC), and exercise capacity (6 minutes walking distance test [6MWD]).

\section{Statistical analysis}

The number of subjects available was greater than required according to sample size calculations (see Supplementary material). We assessed the presence and patterns of missing values and, assuming the missing-at-random hypothesis (ie, missingness conditional on measured patients' characteristics) (see Supplementary material for further detail), ${ }^{26}$ we used multiple imputation (100 imputed datasets) with the method of chained equations. ${ }^{27}$ We compared the characteristics of patients in the complete case to the imputed datasets (Table S2). All analyses were performed using the imputed dataset.

In order to maximize data use we used a repeated measures approach (Figure 1), each patient contributing twice to the analysis. Let $t$ denote each time period (baseline, 6 months, and 12 months). For each patient, we estimated the association between anxiety or depression at $t$ and PA at $t+1$, ie, anxiety or depression at baseline and PA at 6 months, and anxiety or depression at 6 months and PA at 12 months, respectively. In bivariate and multivariable analyses we accounted for the correlation between observations of the same subject.

First, we tested the bivariate associations between anxiety and depression (both categorized according to clinically relevant cut-offs ${ }^{20}$ and as continuous variables) and PA (steps/ day and time in locomotion), using analysis of variance and linear regression, given that PA outcomes were normally distributed. Prior to building multivariable models we used generalized additive model analyses to test whether the shape of the association between anxiety or depression and PA was linear. Then, we used multivariable linear regression to assess the independent effect of anxiety and depression on PA. We tested a list of potential confounders according to a previous systematic review on determinants of PA in COPD ${ }^{1}$ and clinical expertise, including: age, sex, ethnicity, marital status, socio-economic status, working status, smoking status and intensity, study site, $\mathrm{FEV}_{1}, \mathrm{FVC}, \mathrm{TLC}, \mathrm{RV}, \mathrm{RV} / \mathrm{TLC}$, BMI, fat free mass index, dyspnea, 6MWD, total number of comorbidities, cardiovascular disease, diabetes, respiratory

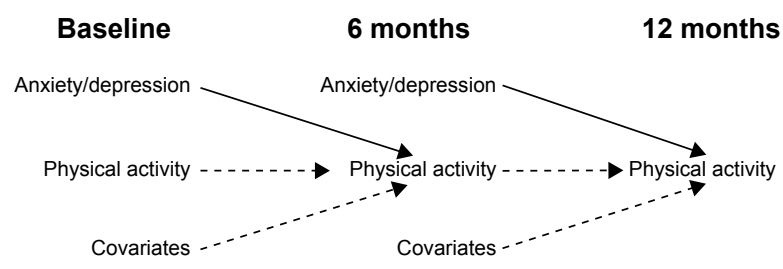

Figure I Approach to assess the effect of anxiety and depression (exposures) at $t$ and physical activity (outcome) at $t+\mathrm{I}$ adjusting for covariates and physical activity at $t$. and non-respiratory drug treatment, CAT score, COPD exacerbations in the previous year, and season of PA measurement. From these variables, we built a "saturated model", which included: i) variables independently related to the exposure or the outcome $(P$-value $<0.05)$, or that modified ( $>10 \%$ change in coefficient) the estimates for the remaining variables and ii) PA at $t$. As a final step, we built a "parsimonious model" using a backward stepwise elimination process from the "saturated model" removing variables (one at a time) if Wald's $P$-value $>0.15 .{ }^{28}$ We tested goodness-of-fit of the final models (Supplementary material).

To identify potential effect modification, we stratified main analyses according to sex, health-related quality of life, COPD spirometric severity, smoking status, comorbidities, exercise capacity, socio-economic status, and BMI (Supplementary material). To test the potential role of COPD exacerbations during follow-up as mediators of the effect of anxiety or depression on PA, we repeated the analyses: i) including "COPD exacerbations during follow-up" as a covariate in multivariable models, and ii) excluding patients who had at least one COPD exacerbation during the follow-up period.

We performed several secondary analyses (Supplementary material) to assess the sensitivity of our estimates to our assumptions regarding biases, as well as to test for model misspecification. Briefly, we repeated analyses: i) using PA at 12 months only (ignoring measures at 6 months), ii) additionally adjusting for season of PA assessment, and iii) using complete case dataset. The analyses were performed using Stata 12.1 (Stata Statistical Software: Release 12; StataCorp LP, College Station, TX, USA) and R 3.0.1 (R Foundation for Statistical Computing, Vienna, Austria).

\section{Results}

A total of 220 patients were included, distributed similarly across study sites (48 [21.8\%] from Athens, 41 [18.6\%] from Edinburgh, 48 [21.8\%] from Leuven, 48 [21.8\%] from London, and 35 [15.9\%] from Groningen). A majority of patients were men (68\%), with a mean (SD) age of 67 (8) years, $\mathrm{FEV}_{1} 57(20) \%$ predicted, RV/TLC 51 (11)\%, BMI $27.1(5.5) \mathrm{kg} / \mathrm{m}^{2}$, and a moderate quality of life (Table 1$)$. The baseline prevalence of suggested and probable anxiety was $19 \%$ and $10 \%$ respectively, and the corresponding prevalence for depression was $16 \%$ and $5 \%$. All followed patients had valid (ie, at least 3 days with at least 10 hours wearing time) data from the accelerometer. The mean (SD) Dynaport wearing time was $880(120) \mathrm{min} /$ day, the mean number of steps was $4,812(3,147)$ and mean time in locomotion was 60 (36) $\mathrm{min} /$ day. During follow-up, 22 (10\%) patients were hospitalized at least once for a COPD exacerbation. 
Table I Baseline sociodemographic, clinical, and functional characteristics of 220 COPD patients from five European sites

\begin{tabular}{|c|c|}
\hline \multirow[t]{2}{*}{ Baseline characteristics } & \multirow{2}{*}{$\begin{array}{l}\text { All patients } \\
\mathrm{n}=\mathbf{2 2 0}\end{array}$} \\
\hline & \\
\hline Age (years), m (SD) & $67(8)$ \\
\hline Sex: male, $\mathrm{n}(\%)$ & $149(68 \%)$ \\
\hline Living alone, n (\%) & $108(49 \%)$ \\
\hline Socio-economic status: ${ }^{\mathrm{a}}$ low (IV and V), n (\%) & $30(14 \%)$ \\
\hline Active worker, $\mathrm{n}(\%)$ & $26(12 \%)$ \\
\hline Smoking status: current, n (\%) & $38(17 \%)$ \\
\hline Pack years, median (P25 to P75) & 47 (3I to 72$)$ \\
\hline Any COPD exacerbation in the previous year, $\mathrm{n}(\%)$ & $88(40 \%)$ \\
\hline mMRC score, median (P25 to P75) & $2(1$ to 2$)$ \\
\hline $\begin{array}{l}\text { Number of chronic diseases (other than COPD), } \\
\text { median (P25 to P75) }\end{array}$ & $2(1$ to 3$)$ \\
\hline Cardiovascular disease, ${ }^{\mathrm{b}} \mathrm{n}(\%)$ & I 18 (54\%) \\
\hline Diabetes, $n(\%)$ & $19(9 \%)$ \\
\hline Musculoskeletal diseases, ${ }^{c} \mathrm{n}(\%)$ & $48(22 \%)$ \\
\hline Osteoporosis, ${ }^{\mathrm{d}} \mathrm{n}(\%)$ & $23(10 \%)$ \\
\hline Body mass index $\left(\mathrm{kg} / \mathrm{m}^{2}\right), \mathrm{m}(\mathrm{SD})$ & $27(5)$ \\
\hline Fat free mass index $\left(\mathrm{kg} / \mathrm{m}^{2}\right), \mathrm{m}(\mathrm{SD})$ & $18(3)$ \\
\hline $\mathrm{FEV}_{1}(\%$ predicted), m (SD) & $57(20)$ \\
\hline \multicolumn{2}{|l|}{ COPD severity stages } \\
\hline I- Mild $\left(F E V_{1} \geq 80 \%\right)$ & $30(14)$ \\
\hline II - Moderate $\left(\mathrm{FEV}_{1}<80 \%\right.$ and $\left.\geq 50 \%\right)$ & I0I (46) \\
\hline III - Severe $(\mathrm{FEV},<50 \%$ and $\geq 30 \%)$ & $69(31)$ \\
\hline IV - Very severe $(\mathrm{FEV},<30 \%)$ & $20(9)$ \\
\hline RV/TLC (\%), m (SD) & $5 I(I I)$ \\
\hline Six minutes walking distance test, m (SD) & $423(130)$ \\
\hline CAT score (0 to 40$)$, median (P25 to P75) & $14(8$ to 21$)$ \\
\hline Probable anxiety (HADS-A $\geq \mathrm{II}), \mathrm{n}(\%)$ & $23(10 \%)$ \\
\hline Probable depression (HADS-D $\geq \mathrm{II}$ ), $\mathrm{n}(\%)$ & $12(5 \%)$ \\
\hline
\end{tabular}

Notes: aSocio-economic status was measured through the UK National Statistics Socio-economic classification. ${ }^{b} \mathrm{C}$ ardiovascular disease was the presence of doctordiagnosed diseases in medical records (ICD9 390-459). Musculoskeletal diseases included connective tissue diseases (ICD9 7/0-739). ${ }^{\mathrm{d}}$ Osteoporosis included osteoporosis (ICD9 733.0) and osteopenia (ICD9 733.90).

Abbreviations: m, mean; SD, standard deviation; P25, 25th percentile; P75, 75th percentile; mMRC, Modified Medical Research Council Dyspnea Scale; FEV expiratory volume in I second; RV/TLC, residual volume to total lung capacity ratio; CAT, COPD assessment test; HADS-A, Hospital Anxiety and Depression Scale-Anxiety; HADS-D, Hospital Anxiety and Depression Scale-Depression; ICD, International Classification of Diseases.

In bivariate analysis, depression symptoms at $t$ were associated with a lower amount of PA at $t+1$ (6 months later) in a linear dose-response shape: 4,752 (2,334), 3,837 $(1,987)$, and $3,446(2,144)$ steps/day in patients with no symptoms, suggested and probable depression, respectively, $P$-trend $=0.02$ (Figure 2 and Table S3 $)$. Corresponding time in locomotion was 59.2 (37.3), 49.1 (31.5), and 39.8 (44.1) $\mathrm{min} /$ day, $P$-trend $=0.01$. The differences of $P A$ across HADS-A categories were not statistically significant.

In the multivariable saturated model (adjusted for age, 6MWD, comorbidities, $\mathrm{FEV}_{1}$, and steps at $t$ ), patients walked 70 steps/day less $(P=0.05)$ for each extra point on the HADS-D score (Table 2). This association persisted in the parsimonious model ( $\beta=-81$ steps/day, $P=0.02$ ). HADS-D score was also significantly associated with less time in locomotion (Table S4). HADS-A score was not statistically associated with any PA outcome in multivariable models.

The effect of HADS-D on steps did not change after stratifying by potential effect modifiers, with two exceptions: i) a stronger association among patients with moderate CAT scores $(\beta=-132, P=0.03)$ compared to patients with lower or higher CAT scores $(\beta=-19, P=0.79$ and $\beta=-26, P=0.73$, respectively), and ii) a stronger association among people with high socio-economic status compared to those with low socio-economic status $(\beta=-88, P=0.02$, vs $-2, P=0.98$, respectively) (Table $\mathrm{S} 5$ ).

The inclusion of COPD exacerbations during follow-up in the model did not modify the estimate of the association between depression and steps either $(\beta=-70$ steps/day per each HADS-D point, $P=0.05$ ) (Table S6). Similarly, the exclusion of patients with any COPD exacerbation during follow up ( $\mathrm{n}=101,46 \%)$ did not modify such estimate $(\beta=-81$, $P=0.15$ ) (Table S6). Sensitivity analyses yielded very similar results (Tables S7-S9). Linear regression goodness-of-fit tests did not reveal any abnormality.

\section{Discussion}

Our study shows that depression symptoms, as measured by the HADS, are prospectively associated with fewer steps per day and less time in locomotion in COPD patients after 6 months. However, no effect of anxiety on PA variables was found.

Previous studies reported a cross-sectional association between depression and PA levels in COPD patients. ${ }^{7-12}$ Our findings move beyond these preliminary findings by testing the directionality of this association. Indeed, presence of depressive symptomatology very quickly seems to negatively impact PA (ie, over a time period of 6 months already), even after adjusting for baseline PA levels. Furthermore, in contrast to previous studies, our study for the first time controlled for a comprehensive set of potential confounders such as lung function, exercise capacity or comorbidities. Moreover, while previous studies focused on the role of depressive disorder, we demonstrated that presence of depressive symptoms impacts PA, a finding that is of major clinical importance. As each extra point in the HADS-D score was associated with a reduction of 81 steps, our results support that COPD management includes the assessment and treatment of mood disorders, even at subclinical stages. We would suggest that further research includes randomized controlled trials of drug and non-drug interventions to 
A

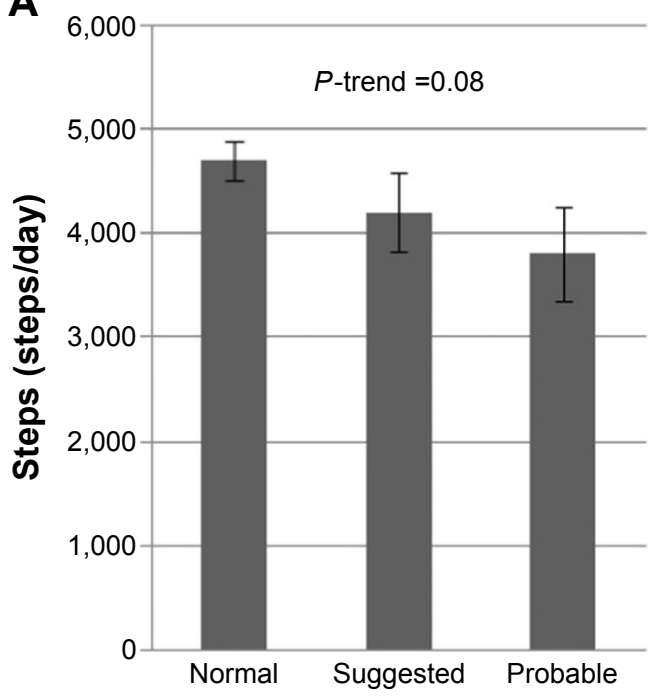

C

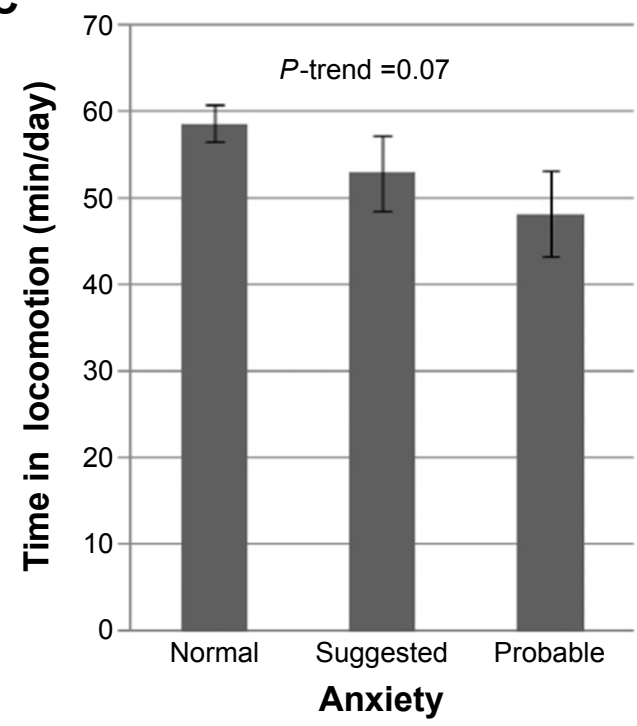

B

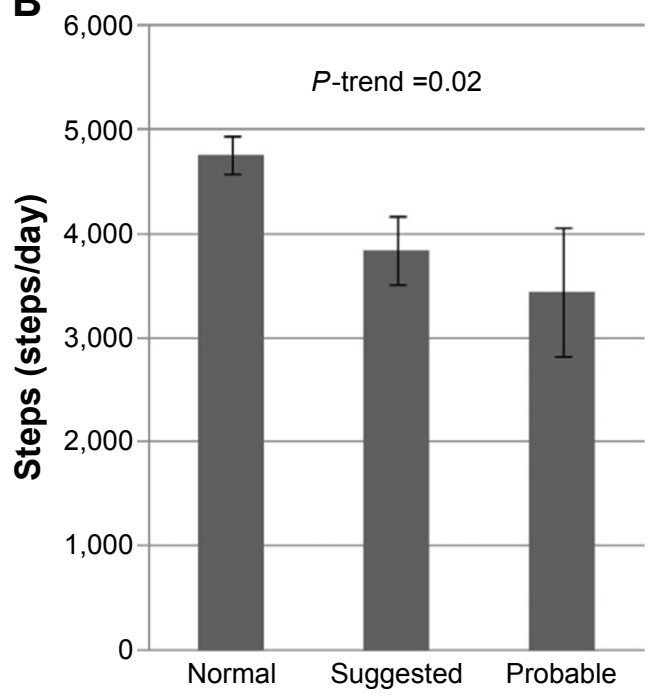

D

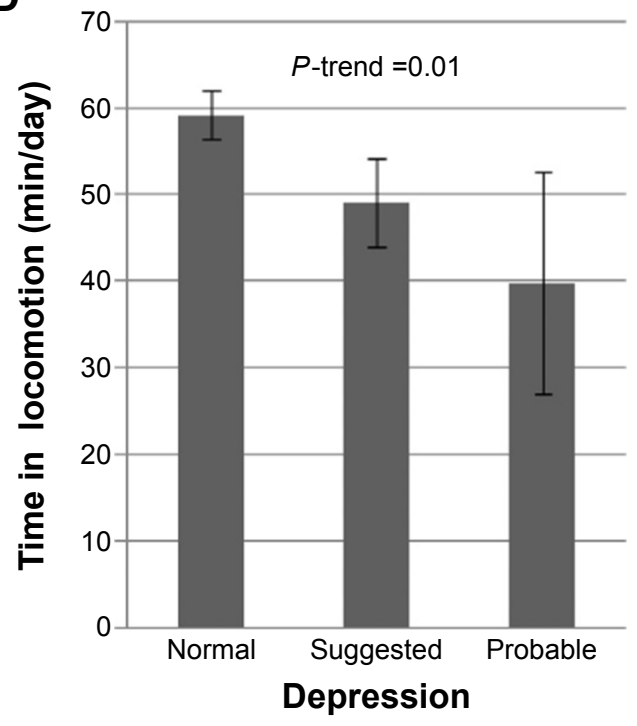

Figure 2 Distribution of steps and time in locomotion at $t+\mathrm{I}$, according to categories of HADS-A and HADS-D at $t$.

Notes: (A) Steps at $t+\mathrm{I}$ across HADS-A categories at $t$; (B) steps at $t+\mathrm{I}$ across HADS-D categories at $t$; (C) time in locomotion $t+\mathrm{I}$ months across HADS-A categories at $t$; and (D) time in locomotion at $t+\mathrm{I}$ across HADS-D categories at $t$. HADS-A score: 0-7 normal, 8-10 suggested anxiety, and $\geq 1 \mathrm{I}$ probable anxiety. HADS-D score: 0-7 normal, 8-10 suggested depression, and $\geq 11$ probable depression.

Abbreviations: HADS-A, Hospital Anxiety and Depression Scale-anxiety; HADS-D, Hospital Anxiety and Depression Scale-Depression.

reduce depression symptoms, since they may be valuable also to improve PA.

Several mechanisms have been proposed that support the plausibility of depression symptoms affecting PA in both COPD and healthy individuals. First, a recent systematic review found a clear association between depression and hospital admissions caused by COPD exacerbations ${ }^{29}$ and an effect of exacerbations on PA levels has also been reported. ${ }^{30}$ However, we could not confirm that the effect of depression on PA is mediated by COPD exacerbations. Second, hormonal changes that appear during depression, including overactivity of the hypothalamo-pituitary-adrenocortical axis ${ }^{31}$ or dysregulation of the autonomous nervous system, ${ }^{32,33}$ have been associated with reduced PA in experimental studies ${ }^{34,35}$ and deserve further scrutiny in COPD patients. Finally, symptoms of depression may exacerbate the perception of dyspnea, ${ }^{36}$ which in turn could reduce PA levels.

The lack of a measurable confounding role of exercise capacity in the association between depression and PA in our study, together with the large body of evidence showing a weak to moderate correlation between exercise capacity and daily PA, ${ }^{37}$ suggests that the clinical effects of depression on COPD patients may be underestimated if only controlled assessment of exercise capacity is performed at the clinical 
Table 2 Crude and adjusted associations between HADS-depression score at $t$ and daily steps at $t+\mathrm{I}$ (linear regression model)

\begin{tabular}{|c|c|c|c|c|c|c|}
\hline \multirow[t]{3}{*}{ Characteristics at $t$} & \multirow{2}{*}{\multicolumn{2}{|c|}{ Crude $^{a}$}} & \multicolumn{4}{|l|}{ Adjusted } \\
\hline & & & \multicolumn{2}{|l|}{ Saturated model ${ }^{b}$} & \multicolumn{2}{|l|}{ Parsimonious model $^{c}$} \\
\hline & $\beta(95 \% \mathrm{Cl})$ & $P$-value & $\beta(95 \% \mathrm{Cl})$ & $P$-value & $\beta(95 \% \mathrm{Cl})$ & $P$-value \\
\hline Constant & N/A & - & $4,690(4,323$ to 5,058$)$ & $<0.01$ & $4,733(4,377$ to 5,089$)$ & $<0.01$ \\
\hline HADS-D (per each extra point) & $-204(-313$ to -96$)$ & $<0.01$ & $-70(-|4|$ to 0$)$ & 0.05 & $-81(-149$ to -12$)$ & 0.02 \\
\hline Age (per each extra year old) & $-86(-13 \mid$ to -42$)$ & $<0.01$ & $-15(-50$ to 19$)$ & 0.37 & - & - \\
\hline $\begin{array}{l}\text { Six minutes walking distance test (6MWD, per } \\
\text { each extra meter) }\end{array}$ & II (8 to I3) & $<0.01$ & $1.7(-0.8$ to 4.2$)$ & 0.17 & - & - \\
\hline$\geq 2$ comorbidities & $-984(-1785$ to -182$)$ & 0.01 & $-332(-833$ to 169$)$ & 0.19 & $-4 \mid 4(-90 \mid$ to 72$)$ & 0.09 \\
\hline $\mathrm{FEV}, \%$ predicted (per each extra percent unit) & $42(21$ to 63$)$ & $<0.01$ & $10(-3$ to 22$)$ & 0.13 & II $(-0.8$ to 23$)$ & 0.06 \\
\hline Steps/day at $t$ (per each extra step) & $0.7(0.6$ to 0.8$)$ & $<0.01$ & $0.6(0.5$ to 0.8$)$ & $<0.01$ & $0.6(0.5$ to 0.8$)$ & $<0.01$ \\
\hline
\end{tabular}

Notes: ${ }^{a}$ Each row is a single model. ${ }^{b}$ The column is a single model. The value of the constant is the number of steps at $t+I$ of a patient with mean HADS-D score, mean age, mean 6MWD, $<2$ comorbidities, mean $\mathrm{FEV}_{1}$ (\% predicted) and mean steps/day at $t$. 'The column is a single model. The value of the constant is the number of steps at $t+1$ of a patient with mean HADS-D score, $<2$ comorbidities, mean FEV (\% predicted) and mean baseline steps/day at $t$.

Abbreviations: $\mathrm{Cl}$, confidence interval; N/A, not applicable; HADS-D, Hospital Anxiety and Depression Scale-Depression; FEV 1 , forced expiratory volume in I second.

or research setting. Further, we found a stronger effect of depression on PA among people with moderate impact on quality of life than in those with low or high impact on quality of life. It could be speculated that patients with a moderate quality of life are those who experience the bigger impact of depressive symptoms on health outcomes, because in milder stages the depression symptoms are lower while in worsened quality of life, patients have reached the acceptance of the disease together with a higher understanding of their limitations. As previous research has reported a strong relationship between depression symptoms and quality of life scores in COPD patients, ${ }^{38,39}$ the interaction between these factors on COPD outcomes should be considered both in clinical practice and research settings.

The role of anxiety in PA levels is unclear because a previous study has found an inverse association (ie, anxiety reduces PA level), ${ }^{12}$ while another study found a positive association (ie, anxiety increases PA level). ${ }^{10}$ Yet, these studies suffer from methodological limitations as previously discussed, including a cross-sectional design, indirect measures of PA, and lack of control for confounders. Our study, overcoming all the aforementioned limitations, found no significant association between anxiety symptoms and PA level in COPD patients. Although several studies have postulated common characteristics between anxiety and depression, both at molecular ${ }^{40}$ and clinical levels ${ }^{41}$ other studies support significant differences between the two. Clinically, levels of psychomotor hyperactivity are increased in anxiety ${ }^{42}$ but reduced in depression, ${ }^{43}$ which could lead to differential effects on PA. Although elucidating these mechanisms is beyond the scope of the present study, our data support the need to further research the pathophysiological differences between the two entities ${ }^{44}$ in order to avoid attributing, a priori, common effects.

The strengths of our study include the exhaustive control for potential confounders, the use of direct methods to assess PA, and the longitudinal design. Limitations of our study are the following. Information bias in the measure of anxiety and depression is possible because the questionnaire is subject to potential mis-classification. However, HADS has been demonstrated to have good psychometric properties. ${ }^{45,46}$ It could be argued that the proportion of patients with HADS anxiety and HADS depression $\geq 11$ was low ( $10 \%$ and $5 \%$, respectively) in comparison with other studies. ${ }^{2-4,47-49}$ This can be due to the fact that we excluded patients with a diagnosis and/or treatment for clinical depression and/or anxiety (to avoid any effect of therapies on PA level). Consequently, it is likely that the association between depression and PA level is higher in the whole COPD population than in our sample. In any case, confirmation of our results in studies using other validated instruments to assess anxiety and depression is warranted. Secondly, it could be argued that our population does not represent the full COPD spectrum and so the results cannot be generalized to all patients. However, it is worth noting that this risk was mitigated by including patients recruited in diverse settings (primary care, hospital, and rehabilitation) and a range of mild to very severe airflow limitation. Anyhow, any difference in the prevalence of depression, anxiety or PA levels with respect to the full COPD population would not have affected the association between them. Third, reverse causation, ie, that PA affects depression symptoms, could have been an alternative interpretation if the study design was cross-sectional. However, our study had a longitudinal prospective design, with anxiety 
and depression being measured at baseline, PA measured 6 months later, and baseline PA having been included as a covariate in the regression models to account for PA history and potential reverse causation. Finally, although the possibility of residual confounding can never be ruled out, to the authors' knowledge, all potential confounders were collected and tested.

\section{Conclusion}

In conclusion, symptoms of depression are prospectively associated with a measurable reduction in PA 6 months later in COPD patients. Whereupon, and since the current management guidelines for patients with COPD include the treatment of comorbidities, our study suggests that the management of symptoms of depression need to be considered, including in subclinical states, to prevent decline in PA over time.

\section{Acknowledgments}

Authors thank the input from the PROactive project Ethics Board, Advisory Board and Patient Input Platform (www. proactivecopd.com/about/advisory-boards). PROactive consortium: Almirall: Nathalie Ivanoff; AstraZeneca AB: Niklas Karlsson, Solange Corriol-Rohou; British Lung Foundation, UK: Ian Jarrod; Boehringer Ingelheim: Damijen Erzen; Chiesi Farmaceutici S.A.: Caterina Brindicci, Tim Higenbottam, Mario Scuri; Choice Healthcare Solution, UK: Paul McBride; European Respiratory Society, Lausanne: Nadia Kamel; GlaxoSmithKline: Margaret Tabberer; Katholieke Universiteit Leuven: Thierry Troosters, Fabienne Dobbels; Municipal Institute of Medical Research, Barcelona: Judith Garcia-Aymerich; the Netherlands Asthma Foundation, Amersfoort: Pim de Boer; Novartis: Karoly Kulich, Alastair Glendenning; Pfizer: Katja Rudell, Frederick J Wilson; Royal Brompton and Harefield NHS Foundation Trust: Michael I Polkey, Nick S Hopkinson; Thorax Research Foundation, Athens: Ioannis Vogiatzis; UCB: Enkeleida Nikai; University Medical Center, Groningen: Thys van der Molen, Corina De Jong; University of Edinburgh, Old College South Bridge: Roberto A Rabinovich, Bill MacNee; University of Zurich, Zurich: Milo A Puhan, Anja Frei.

Supported by the European Commission Innovative Medicines Initiative Joint Undertaking (IMI JU number 115011). No involvement of funding source in study design; in the collection, analysis, and interpretation of data; in the writing of the report; nor in the decision to submit the article for publication. Work at the Brompton was supported by the NIHR Respiratory Disease Biomedical Research Unit at the Royal Brompton and Harefield NHS Foundation Trust and Imperial College London which part-fund Michael I Polkey's salary. The views expressed in this publication are those of the authors and not necessarily those of the NHS, The National Institute for Health Research or the Department of Health. The PROactive consortium approved the final version of the manuscript.

\section{Author contributions}

JGA takes responsibility for (is the guarantor of) the content of the manuscript, including the data and analysis. IDE and JGA led the study, performed the statistical analysis, and drafted the manuscript. All authors contributed to conception, hypotheses delineation, design of the study, acquisition of the data or analysis and interpretation of results; critically revising the article; approved the final version to be published; and agree to be accountable for all aspects of the work.

\section{Disclosure}

Michael I Polkey's institution received payment $(<\$ 5,000)$ for the participation in an advisory board organized by Boehringer Ingelheim. Thierry Troosters has provided consultancy or given sponsored talks around the topic to Boehringer Ingelheim, Novartis, and GSK (amounts paid to the institution $<5,000 €)$. Niklas Karlsson is employed by AstraZeneca. Iván Dueñas-Espín, Heleen Demeyer, Elena Gimeno-Santos, Nicholas S Hopkinson, Roberto A Rabinovich, Fabienne Dobbels, and Judith Garcia-Aymerich have no conflicts of interest to disclose.

\section{References}

1. Gimeno-Santos E, Frei A, Steurer-Stey C, et al. Determinants and outcomes of physical activity in patients with COPD: a systematic review. Thorax. 2014;69(8):731-739.

2. Al-shair K, Dockry R, Mallia-Milanes B, et al. Depression and its relationship with poor exercise capacity, BODE index and muscle wasting in COPD. Respir Med. 2009;103(10):1572-1579.

3. de Voogd JN, Wempe JB, Koëter GH, et al. Depressive symptoms as predictors of mortality in patients with COPD. Chest. 2009;135(3): 619-625.

4. Atlantis E, Fahey P, Cochrane B, Smith S. Bidirectional associations between clinically relevant depression or anxiety and COPD: a systematic review and meta-analysis. Chest. 2013;144(3):766-777.

5. Panagioti M, Scott C, Blakemore A, Coventry PA. Overview of the prevalence, impact, and management of depression and anxiety in chronic obstructive pulmonary disease. Int J Chron Obstruct Pulmon Dis. 2014;9:1289-1306.

6. Cooper CB. The connection between chronic obstructive pulmonary disease symptoms and hyperinflation and its impact on exercise and function. Am J Med. 2006;119(10 Suppl 1):21-31.

7. Weaver TE, Narsavage GL. Physiological and psychological variables related to functional status in chronic obstructive pulmonary disease. Nurs Res. 1992;41(5):286-291. 
8. Yeh ML, Chen HH, Liao YC, Liao WY. Testing the functional status model in patients with chronic obstructive pulmonary disease. $J A d v$ Nurs. 2004;48(4):342-350.

9. Wall MP. Predictors of functional performance in community-dwelling people with COPD. J Nurs Scholarsh. 2007;39(2):222-228.

10. Nguyen HQ, Fan VS, Herting J, et al. Patients with COPD with higher levels of anxiety are more physically active. Chest. 2013;144(1): 145-151.

11. Venkata A, DeDios A, ZuWallack R, Lahiri B. Are depressive symptoms related to physical inactivity in chronic obstructive pulmonary disease? J Cardiopulm Rehabil Prev. 2012;32(6):405-409.

12. Eisner MD, Blanc PD, Yelin EH, et al. Influence of anxiety on health outcomes in COPD. Thorax. 2010;65(3):229-234.

13. Altenburg WA, Bossenbroek L, de Greef MH, et al. Functional and psychological variables both affect daily physical activity in COPD: a structural equations model. Respir Med. 2013;107(11):1740-1747.

14. Miravitlles M, Cantoni J, Naberan K. Factors associated with a low level of physical activity in patients with chronic obstructive pulmonary disease. Lung. 2014;192(2):259-265.

15. Weldam SW, Lammers JW, Decates RL, Schuurmans MJ. Daily activities and health-related quality of life in patients with chronic obstructive pulmonary disease: psychological determinants: a cross-sectional study. Health Qual Life Outcomes. 2013;11:190.

16. Schüz N, Walters JA, Cameron-Tucker H, et al. Patient Anxiety and Depression Moderate the Effects of Increased Self-management Knowledge on Physical Activity: A Secondary Analysis of a Randomised Controlled Trial on Health-Mentoring in COPD. COPD. 2015; 12(5):502-509.

17. Ferrari P, Friedenreich C, Matthews CE. The role of measurement error in estimating levels of physical activity. Am J Epidemiol. 2007; 166(7):832-840

18. Gimeno-Santos E, Raste Y, Demeyer H, et al. The PROactive instruments to measure physical activity in patients with chronic obstructive pulmonary disease. Eur Respir J. 2015;46(4):988-1000.

19. Zigmond AS, Snaith RP. The hospital anxiety and depression scale. Acta Psychiatr Scand. 1983;67(6):361-370.

20. Snaith RP. The Hospital Anxiety And Depression Scale. Health Qual Life Outcomes. 2003;1:29.

21. Van Remoortel H, Raste Y, Louvaris Z, et al. Validity of six activity monitors in chronic obstructive pulmonary disease: a comparison with indirect calorimetry. PLoS One. 2012;7(6):e39198.

22. Rabinovich RA, Louvaris Z, Raste $Y$, et al. Validity of physical activity monitors during daily life in patients with COPD. Eur Respir J. 2013; 42(5):1205-1215.

23. Trost SG, McIver KL, Pate RR. Conducting accelerometer-based activity assessments in field-based research. Med Sci Sports Exerc. 2005;37(11 Suppl):531-543.

24. Heil DP, Brage S, Rothney MP. Modeling physical activity outcomes from wearable monitors. Med Sci Sports Exerc. 2012; 44(1 Suppl 1):S50-S60.

25. Demeyer H, Burtin C, Van Remoortel H, et al. Standardizing the analysis of physical activity in patients with COPD following a pulmonary rehabilitation program. Chest. 2014;146(2):318-327.

26. van Buuren S, Boshuizen HC, Knook DL. Multiple imputation of missing blood pressure covariates in survival analysis. Stat Med. 1999; 18(6):681-694.

27. Donders AR, van der Heijden GJ, Stijnen T, Moons KG. Review: a gentle introduction to imputation of missing values. J Clin Epidemiol. 2006; 59(10):1087-1091.

28. Hosmer DW, Lemeshow S. Applied logistic regression. 2nd ed. New York: Wiley; 2000.

29. Pooler A, Beech R. Examining the relationship between anxiety and depression and exacerbations of COPD which result in hospital admission: a systematic review. Int J Chron Obstruct Pulmon Dis. 2014;9: 315-330.
30. Pitta F, Troosters T, Probst VS, et al. Physical activity and hospitalization for exacerbation of COPD. Chest. 2006;129(3):536-544.

31. Rubin RT. Pharmacoendocrinology of major depression. Eur Arch Psychiatry Neurol Sci. 1989;238(5-6):259-267.

32. Esler M, Turbott J, Schwarz R, et al. The peripheral kinetics of norepinephrine in depressive illness. Arch Gen Psychiatry. 1982;39(3): 295-300.

33. Veith RC, Lewis N, Linares OA, et al. Sympathetic nervous system activity in major depression. Basal and desipramine-induced alterations in plasma norepinephrine kinetics. Arch Gen Psychiatry. 1994; 51(5):411-422.

34. Droste SK, Gesing A, Ulbricht S, et al. Effects of long-term voluntary exercise on the mouse hypothalamic-pituitary-adrenocortical axis. Endocrinology. 2003;144(7):3012-3023.

35. Mueller PJ. Exercise training and sympathetic nervous system activity: evidence for physical activity dependent neural plasticity. Clin Exp Pharmacol Physiol. 2007;34(4):377-384.

36. [No authors listed]. Dyspnea. Mechanisms, assessment, and management: a consensus statement. American Thoracic Society. Am J Respir Crit Care Med. 1999;159(1):321-340.

37. Di Marco F, Santus P, Sotgiu G, Blasi F, Centanni S. Does Improving Exercise Capacity and Daily Activity Represent the Holistic Perspective of a New COPD Approach? COPD. 2015;12(5):575-581.

38. Miravitlles M, Molina J, Quintano JA, et al. Factors associated with depression and severe depression in patients with COPD. Respir Med. 2014;108(11):1615-1625.

39. Balcells E, Gea J, Ferrer J. Factors affecting the relationship between psychological status and quality of life in COPD patients. Heal Qual Life Outcomes. 2010;8:108.

40. Kendler KS. Major depression and generalised anxiety disorder. Same genes, (partly) different environments - revisited. Br J Psychiatry Suppl. 1996;(30):68-75.

41. Kessler RC, Nelson CB, McGonagle KA, et al. Comorbidity of DSM-III-R major depressive disorder in the general population: results from the US National Comorbidity Survey. Br J Psychiatry Suppl. 1996; (30):17-30.

42. Sakamoto N, Yoshiuchi K, Kikuchi H, et al. Panic disorder and locomotor activity. Biopsychosoc Med. 2008;2:23.

43. Buyukdura JS, McClintock SM, Croarkin PE. Psychomotor retardation in depression: biological underpinnings, measurement, and treatment. Prog Neuropsychopharmacol Biol Psychiatry. 2011;35(2):395-409.

44. Hendriks SM, Licht CM, Spijker J, et al. Disorder-specific cognitive profiles in major depressive disorder and generalized anxiety disorder. BMC Psychiatry. 2014;14:96.

45. Mykletun A, Stordal E, Dahl AA. Hospital Anxiety and Depression (HAD) scale: factor structure, item analyses and internal consistency in a large population. Br J Psychiatry. 2001;179:540-544.

46. Bjelland I, Dahl AA, Haug TT, Neckelmann D. The validity of the Hospital Anxiety and Depression Scale: an updated literature review. J Psychosom Res. 2002;52(2):69-77.

47. Kunik ME, Roundy K, Veazey C, et al. Surprisingly high prevalence of anxiety and depression in chronic breathing disorders. Chest. 2005; 127(4):1205-1211.

48. Maurer J, Rebbapragada V, Borson S, et al. Anxiety and depression in COPD: current understanding, unanswered questions, and research needs. Chest. 2008;134(4 Suppl):43S-56S.

49. Hill K, Geist R, Goldstein RS, Lacasse Y. Anxiety and depression in end-stage COPD. Eur Respir J. 2008;31(3):667-677. 
International Journal of COPD

\section{Publish your work in this journal}

The International Journal of COPD is an international, peer-reviewed journal of therapeutics and pharmacology focusing on concise rapid reporting of clinical studies and reviews in COPD. Special focus is given to the pathophysiological processes underlying the disease, intervention programs, patient focused education, and self management protocols.

This journal is indexed on PubMed Central, MedLine and CAS. The manuscript management system is completely online and includes a very quick and fair peer-review system, which is all easy to use. Visit http://www.dovepress.com/testimonials.php to read real quotes from published authors 